\title{
Pharmacokinetics of Midazolam in Critically Ill Patients Receiving Continuous Hemodiafiltration using a Polymethyl Methacrylate and/or Polysulfone Membrane
}

\author{
Kiyotaka Hirata $^{* 1,2}$, Yoshiaki Matsumoto ${ }^{2}$, Kanae Ohno ${ }^{2}$, \\ Masaaki Hirano ${ }^{1}$ and Yasuhiro Yamamoto ${ }^{3}$ \\ Department of Pharmacy, Nippon Medical School, Hospital ${ }^{1}$ \\ Department of Clinical Pharmacology and Toxicology, Showa Pharmaceutical University ${ }^{2}$ \\ Department of Emergency and Critical Care Medicine, Nippon Medical School, Hospital ${ }^{3}$ \\ $\left[\begin{array}{l}\text { Received June 1, } 2004 \\ \text { Accepted November 8, } 2004\end{array}\right]$
}

\begin{abstract}
The objectives of this study were to evaluate the risk of the accumulation of midazolam and its active metabolites in critically ill patients in an intensive care unit (ICU) being treated by continuous hemodiafiltration (CHDF) using a polymethyl methacrylate (PMMA) and/or a polysulfone (PS) membrane and the influence of CHDF on the pharmacokinetics of midazolam in these patients. The serum and ultrafiltration liquid concentrations of midazolam and its active metabolites were measured by high-performance liquid chromatography to study the pharmacokinetics of midazolam and its active metabolites in seven patients with renal failure who were being treated by CHDF using a PMMA and/or a PS membrane. There was no progressive accumulation of midazolam or 1-hydroxymidazolam, though high levels of 1-hydroxymidazolam glucuronide were noted. The extraction rates for these 3 substances by CHDF using a PMMA and a PS membrane were 1.3 $\%, 3.0 \%$ and $13.4 \%$, and $5.0 \%, 4.8 \%$ and $74.4 \%$, respectively. A positive correlation was observed between the 1 hydroxymidazolam glucuronide concentration and serum creatinine concentration for CHDF using the PMMA membrane $\left(\mathrm{r}^{2}\right.$ $=0.8223)$, while there was no correlation between these concentrations when the PS membrane was used $\left(r^{2}=0.0361\right)$. These results show that CHDF using a PMMA membrane did not affect the elimination of 1-hydroxymidazolam glucuronide but it was affected when the PS membrane was used. The risk of accumulation of 1-hydroxymidazolam glucuronide and the need for prolonged sedation should be therefore be taken into account in drug dose adjustment for patients undergoing CHDF.
\end{abstract}

Key words — pharmacokinetics, midazolam, continuous hemodiafiltration, polymethyl methacrylate, polysulfone membrane

\section{Introduction}

For intensive-care patients suffering from multiple organ failure, blood purification therapy (BPT) is an important supportive extracorporeal renal replacement therapy, and acute renal failure is a common complication in critically ill patients. Currently in Japan, the preferred method for BPT in patients with acute renal failure is intermittent hemodialysis and continuous hemodiafiltration (CHDF). CHDF is a recently developed BPT technique and is, at present, widely used in the treatment of critically ill patients with acute renal failure. In such patients, CHDF was reported to provide advantages over intermittent hemodialysis because of a better control of fluid balance, better metabolic control, increased hemodynamic stability, allowance of full nutritional support, and a shorter duration of the episode of renal failure and intensive care unit (ICU) admission ${ }^{1,2)}$.

Since the 1980s, midazolam has been frequently used worldwide for the long-term sedation of patients receiving mechanical ventilation, particularly in ICUs, because of its short elimination half-life, water solubility, and a lack of serious adverse effects ${ }^{3,4}$. However, data concerning the influence of CHDF on midazolam and its active metabolites have not been evaluated. Because of the prolonged sedation observed in severe renal failure patients after receiving a longterm infusion of midazolam, the pharmacokinetic studies of midazolam in critically ill patients treated by CHDF are considered important. Recently, many types of membrane for CHDF have been developed. However, few data on the 
clearance of midazolam and its active metabolites using these various membranes were reported. This study was designed to evaluate the influence of CHDF using polymethyl methacrylate (PMMA) and a polysulfone (PS) membrane on the pharmacokinetics of midazolam and its active metabolites in ICU patients.

\section{Materials and Methods}

\section{Patients and administration of midazolam}

This clinical study was carried out on ICU patients. All patients were intubated, mechanically ventilated and sedated by a continuous infusion of midazolam. These patients underwent CHDF to maintain their fluid and electrolyte balance. The study protocol was approved by our institutional review board, and informed consent for participation was obtained from each patient or the patient's family. The clinical features of the patients, the durations of infusion, and infusion rates of midazolam are shown in Table 1. Sedation was started after the patients had been attached to a mechanical ventilator, and midazolam was administered until mechanical ventilation was tolerated comfortably. Infusion rate was determined by the clinician responsible for each patient and was not influenced by the investigators. No other benzodiazepines were administered during the study period.

\section{2. $C H D F$}

Vascular access for CHDF was obtained by the insertion of an 11-Fr double lumen catheter (Argyle, 6011 A 20-W) in either the femoral or the right internal jugular vein. CHDF was performed using a hollow-fiber membrane (HEMOFEEL ${ }^{\circledR}$ CH-1.0 L, Toray Medical Inc.) composed of a PMMA membrane and/or a high-flux capillary membrane (HEMOFEEL ${ }^{\circledR}$ SH-1.0, Toray Medical Inc.) composed of a PS membrane. Replacement fluid (Sublood B ${ }^{\circledR}$, Fuso, Japan) was delivered after the membrane into the venous limb of the circuit at a rate appropriate for each patient's requirement. Blood was pumped through the membrane at a rate between 10 and $15 \mathrm{~mL} / \mathrm{kg} / \mathrm{min}$. Standard dialysate was delivered countercurrently to the blood flow at a rate of $0.5 \mathrm{~L} /$ $\mathrm{h}$ by a volumetric pump. Ultradiafiltrate flow was set at a rate of between 0.8 and $1.3 \mathrm{~L} / \mathrm{h}$. Nafamostat mesilate was administered as an anticoagulant, and activated coagulation time was maintained between 150 and $180 \mathrm{~s}$ throughout the procedure. The ultrafiltration rate of the filters was $32.5 \pm$ $13.7 \mathrm{~mL} / \mathrm{mmHg}$ per hour at $2 \mathrm{~h}$ into the CHDF and did not change significantly over $24 \mathrm{~h}$.

\section{Sampling and drug analysis}

Blood samples were collected through the sampling ports of the CHDF circuit before and after the filter, and urine samples and ultradiafiltrate samples were also collected for the steady-state assay of midazolam and its active metabolites. Blood samples were collected every day during the study period. Serum was separated and stored at $-80^{\circ} \mathrm{C}$ until analysis. The concentrations of serum unconjugated midazolam and 1-hydroxymidazolam were determined by highperformance liquid chromatography (HPLC). The HPLC equipment consisted of the following:an LC-10AD constantflow pump (Shimadzu), an SIL-10Axl auto sample injector (Shimadzu), a TSK-GEL Octyl-80 TS column (150 $\mathrm{mm} \times 4.6$ $\mathrm{mm}$; Tosoh), and an SPD-10A ultraviolet detector(242 nm; Shimadzu). The mobile phase was an acetonitrile-methanol $60 \mathrm{mM}$ phosphate buffer (pH 6.5) $(30: 15: 55 \mathrm{v} / \mathrm{v})$ with a flow rate of $1 \mathrm{~mL} / \mathrm{min}$. Clotiazepam, the internal standard, was prepared by dissolving in methanol and diluting with distilled water. The samples were extracted following the method described by Boukhabza et al. ${ }^{5)}$ with a slight modification. Test solutions were injected onto the HPLC column. Linear regression, which was obtained by plotting the peak height ratios of midazolam and 1-hydroxymida-zolam to the internal standard against the added concentrations, was used to determine the slope and intercept. Total drug concentration was measured in the same samples after enzymatic hydrolysis with $\beta$-glucuronidase for $5 \mathrm{~h}$. The difference between total and unconjugated drug concentrations was taken

Table 1. Demographic Data.

\begin{tabular}{|c|c|c|c|c|c|c|c|c|}
\hline \multirow[t]{2}{*}{ Patient } & \multirow[t]{2}{*}{ Sex } & \multirow{2}{*}{$\begin{array}{l}\text { Age } \\
(y)\end{array}$} & \multirow{2}{*}{$\begin{array}{l}\text { BW } \\
(\mathrm{kg}) \\
\end{array}$} & \multirow[t]{2}{*}{ Diagnosis } & \multicolumn{2}{|c|}{ MDZ } & \multirow{2}{*}{$\begin{array}{l}\text { Albumin } \\
\text { (g/dL) }\end{array}$} & \multirow[t]{2}{*}{ Membrane } \\
\hline & & & & & \multicolumn{2}{|c|}{ Infusion Rate Duration } & & \\
\hline 1 & M & 74 & 50 & pneumonia & $0.03-0.2$ & 8 & $2.1-3.3$ & PMMA \\
\hline 2 & $\mathrm{~F}$ & 45 & 50 & $\mathrm{AP}$ & $0.2-0.3$ & 6 & 2.1-3.1 & PMMA, PS \\
\hline 3 & $M$ & 73 & 53 & sepsis & $0.1-0.19$ & 13 & $2.2-3.1$ & PS \\
\hline 4 & M & 65 & 56 & $A P$ & 0.45 & 17 & 2.4-3.3 & PMMA \\
\hline 5 & M & 59 & 75 & burn & $0.27-0.4$ & 9 & $2.0-3.2$ & PMMA, PS \\
\hline 6 & M & 39 & 85 & $\mathrm{AP}$ & $0.18-0.35$ & 8 & $2.1-3.7$ & PMMA, PS \\
\hline 7 & $M$ & 48 & 80 & AP & $0.25-0.38$ & 8 & $2.7-3.6$ & PMMA, PS \\
\hline Mean $\pm s$ & & \multicolumn{3}{|c|}{$57.6 \pm 13.964 .1 \pm 15.2$} & \multicolumn{3}{|c|}{$9.9 \pm 3.8$} & \\
\hline
\end{tabular}

MDZ: midazolam, PMMA: polymethyl methacrylate, PS: polysulfone, AP:acute pancreatitis 
as conjugated drug concentration. Midazolam was provided by Yamanouchi (Tokyo, Japan), and clotiazepam by Yoshitomi (Osaka, Japan). 1-Hydroxymidazolam was obtained from UFC Ltd. (Manchester, England) and $\beta$-glucuronidase from Boehringer Mannheim. Other chemicals used were of analytical or reagent grade. The quantitation limit was $8 \mathrm{ng} /$ $\mathrm{mL}$ for both midazolam and 1-hydroxymidazolam.

\section{Pharmacokinetic analysis}

Extraction rate (ER) was calculated from the following equation : $\mathrm{ER}(\%)=(\mathrm{Cbi}-\mathrm{Cbo}) \times 100 / \mathrm{Cbi}$, where $\mathrm{Cbi}$ represents the concentration of a drug in the blood going in and Cbo the concentration of a drug in the blood flowing out. Clearance was calculated from the following equation ${ }^{6)}: \mathrm{CL}$ $=\mathrm{Qbo} \times(\mathrm{Cbi}-\mathrm{Cbo}) / \mathrm{Cbi}+\mathrm{QF}$, where $\mathrm{Qbo}$ is the blood flow rate out, $\mathrm{Cbi}$ is the concentration of drug in the blood going in, Cbo is the concentration of a drug in the blood flowing out, and QF is the ultrafiltration flow rate out. The concentrations in serum obtained after continuous intravenous infusions were used to calculate the elimination rate constant (ke) of midazolam based on a one-compartment open model with first-order kinetics. The elimination halflife $(\mathrm{t} 1 / 2)$ was calculated from ke using the equation $: t 1 / 2$ $=0.693 / \mathrm{ke}$.

\section{Results}

Seven patients (6 males and 1 female) receiving a longterm infusion of midazolam were studied. Their clinical features, durations and infusion rates of midazolam are shown in Table 1. The continuous infusion of midazolam at 0.03 $0.45 \mathrm{mg} / \mathrm{kg}$ body weight $/ \mathrm{h}$ was maintained for long-term sedation. The concentrations of midazolam, 1-hydroxymidazolam, and 1-hydroxymidazolam glucuronide at each sampling port for patients 1 and 2 during CHDF are shown in
Fig. 1 and Fig. 2, respectively. The extraction rates (\%) of CHDF using PMMA (patient 1) and PS (patient 2) for midazolam, 1-hydroxymidazolam, and 1-hydroxymidazolam glucuronide were 1.3,3.0 and 13.4 and 5.0,4.8 and 74.4, respectively. The clearance $(\mathrm{mL} / \mathrm{min}$ ) during CHDF using PMMA and PS for the three substances was 15.3,13.9 and 18.7 and 15.9,15.7 and 58.5, respectively. Fig. 3 shows the correlation between 1-hydroxymidazolam glucuronide concentration and renal functions in the patients during CHDF using PMMA. A positive correlation between 1-hydroxymidazolam glucuronide concentration and serum creatinine level was observed (least-squares linear regression ; $\mathrm{r}^{2}=0.8223$ ). In contrast, for the patients receiving CHDF using PS, 1-hydroxymidazolam glucuronide concentration did not correlate with serum creatinine level (least-squares linear regression; $\mathrm{r}^{2}=$ 0.0361 (Fig. 4). Likewise, midazolam and 1-hydroxymidazolam concentrations did not correlate with serum creatinine level in the patients during CHDF using PMMA $\left(\mathrm{r}^{2}=\right.$ $0.2763, r^{2}=0.0638$, respectively) and using PS $\left(r^{2}=0.0097\right.$, $\mathrm{r}^{2}=0.3495$, respectively). The serum midazolam, 1-hydroxymidazolam, and 1-hydroxymidazolam glucuronide concentration time profiles and clinical data of patients 6 and 7 receiving CHDF are presented in Fig. 5 and Fig. 6. The calculated terminal half-lives of midazolam, 1-hydroxymi-dazolam, and 1-hydroxymidazolam glucuronide in serum in patients 6 and 7 after the cessation of midazolam infusion during CHDF using PS were in the ranges of 7.2-31.8 h, 9.6-27.4 h, and 7.6-527.6 h, respectively. Three days after the cessation of midazolam infusion, the concentrations of midazolam, 1hydroxymidazolam, and 1-hydroxymidazolam glucuronide decreased to below the therapeutic range in patient 6 , although the concentration of 1-hydroxymidazolam glucuronide remained extremely high in patient 7 who showed deteriorating renal functions.

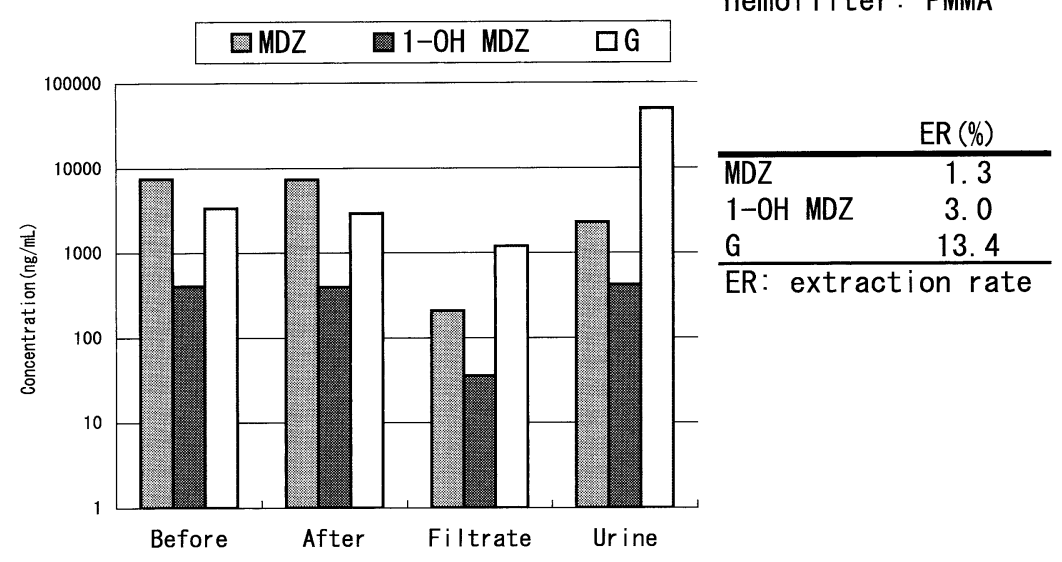

Fig. 1. Concentrations of Midazolam and Active Metabolites at Each Sampling Port in Patient 1 (Before= sampling port before placement of the filter, After $=$ sampling port after placement of the filter, Filtrate $=$ ultradiafiltrate, $\mathrm{MDZ}=$ midazolam, $1-\mathrm{OH} \mathrm{MDZ}=$ 1-hydroxymidazolam, $\mathrm{G}=1$-hydroxymidazolam glucuronide). 


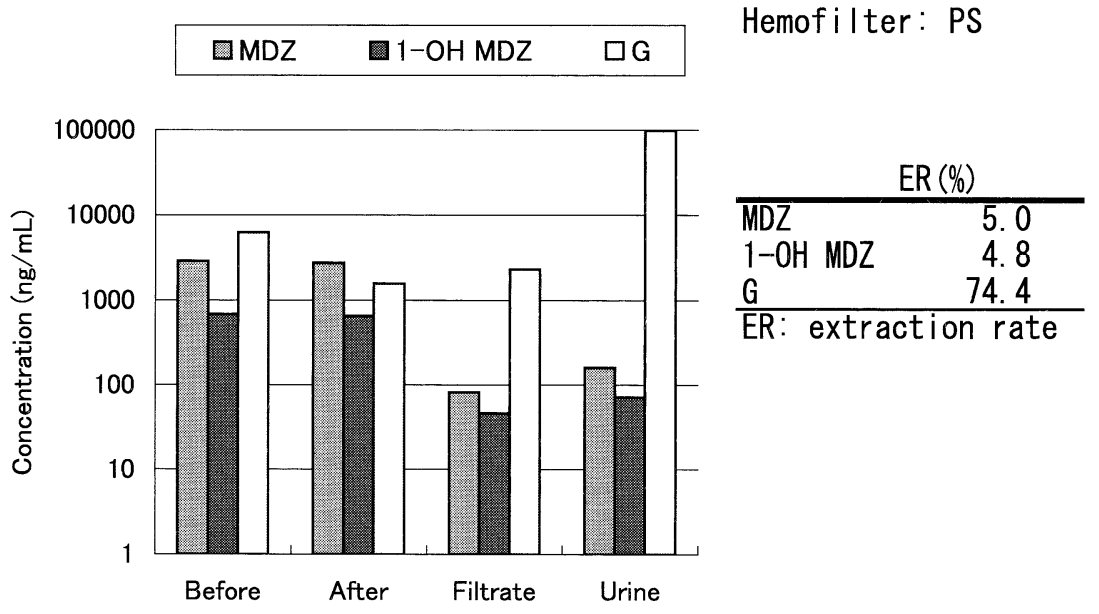

Fig. 2. Concentrations of Midazolam and Active Metabolites at Each Sampling Port in Patient 2 (Before= sampling port before placement of the filter, After=sampling port after placement of the filter, Filtrate $=$ ultradiafiltrate, $\mathrm{MDZ}=$ midazolam, $1-\mathrm{OH} \mathrm{MDZ}=$ 1-hydroxymidazolam, $\mathrm{G}=1$-hydroxymidazolam glucuronide).

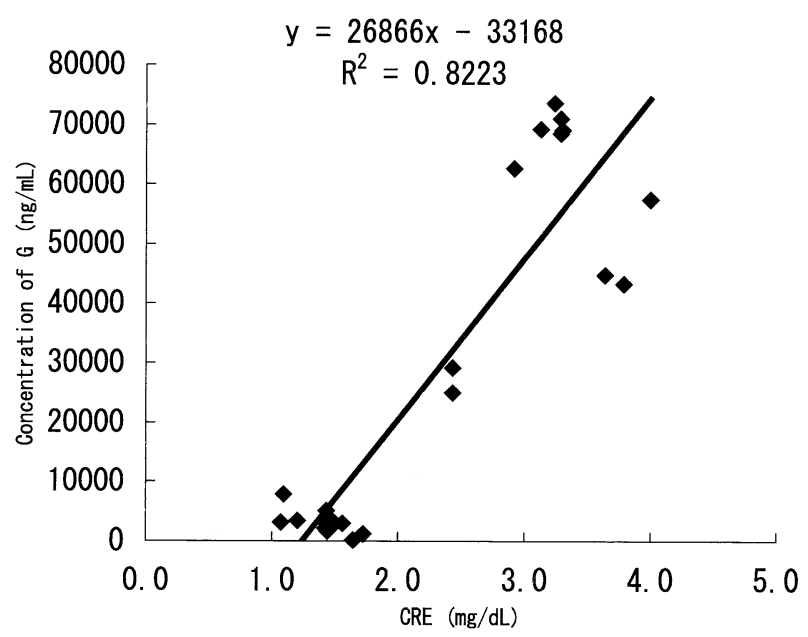

Fig. 3. Correlation between 1-hydroxymidazolam Glucuronide Concentration and Renal Functions in Patients during CHDF using PMMA (CRE $=$ serum creatinine, $\mathrm{G}=1$-hydroxymidazolam glucuronide).

\section{Discussion}

Midazolam is a water-soluble benzodiazepine with sedative-hypnotic, anticonvulsant, muscle relaxant and anxiolytic effects ${ }^{7}$. Midazolam is rapidly eliminated from the body by its transformation to three metabolites, namely, 1hydroxymidazolam ( $63 \%$ of the potency of midazolam), 4hydroxymidazolam (inactive) and 1-hydroxymidazolam glucuronide $(6 \% \text { of the potency of midazolam })^{8)}$. Except for $4-$ hydroxymidazolam, these compounds induce prolonged coma and respiratory suppression. The first step in the metabolism of midazolam is hydroxylation by the hepatic cyto-

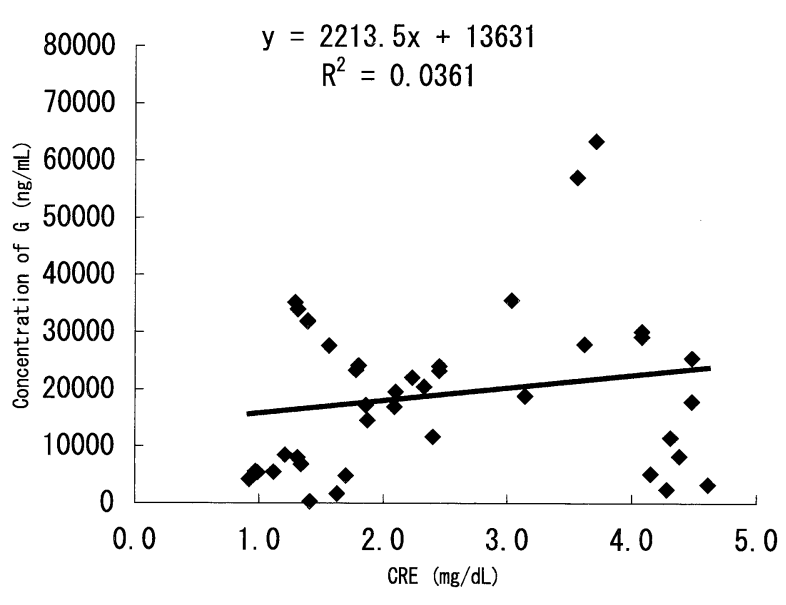

Fig. 4. Correlation between 1-hydroxymidazolam Glucuronide Concentration and Renal Functions in Patients during $\mathrm{CHDF}$ using PS $(\mathrm{CRE}=$ serum creatinine, $\mathrm{G}=1$-hydroxymidazolam glucuronide).

chrome P $4503 \mathrm{~A} 4$, followed by glucuronidation in the liver, and then elimination from the kidneys. However, little is known about the pharmacokinetics and removal of unconjugated and glucuronidated 1-hydroxymidazolam during CHDF. The aim of this study was to evaluate the pharmacokinetics of midazolam in seven ICU patients receiving CHDF.

The elimination half-life of midazolam is about $3 \mathrm{~h}$, although a wide variation has been observed in healthy volunteers and patients ${ }^{9,10)}$. As in previous studies, we observed that the elimination half-life of midazolam was prolonged in renal failure patients after the cessation of midazolam infusion even during CHDF. Although the serum concentrations 


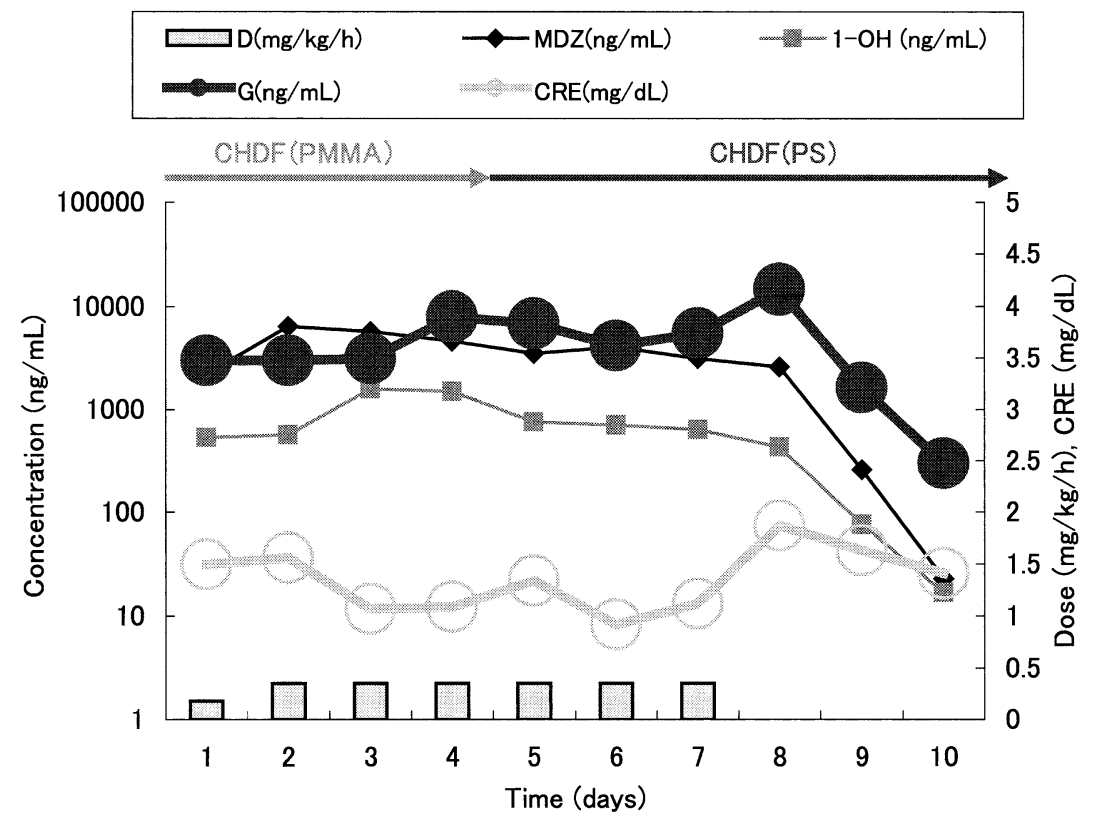

Fig. 5. Serum Midazolam and Active Metabolites Concentration-time Profiles and Clinical Data of Patient 6 Receiving CHDF ( $D=$ infusion rate, $\mathrm{MDZ}=$ midazolam, $1-\mathrm{OH} \mathrm{MDZ}=1$-hydroxymidazolam, $\mathrm{G}=1$-hydroxymidazolam glucuronide, $\mathrm{CRE}=$ serum creatinine).

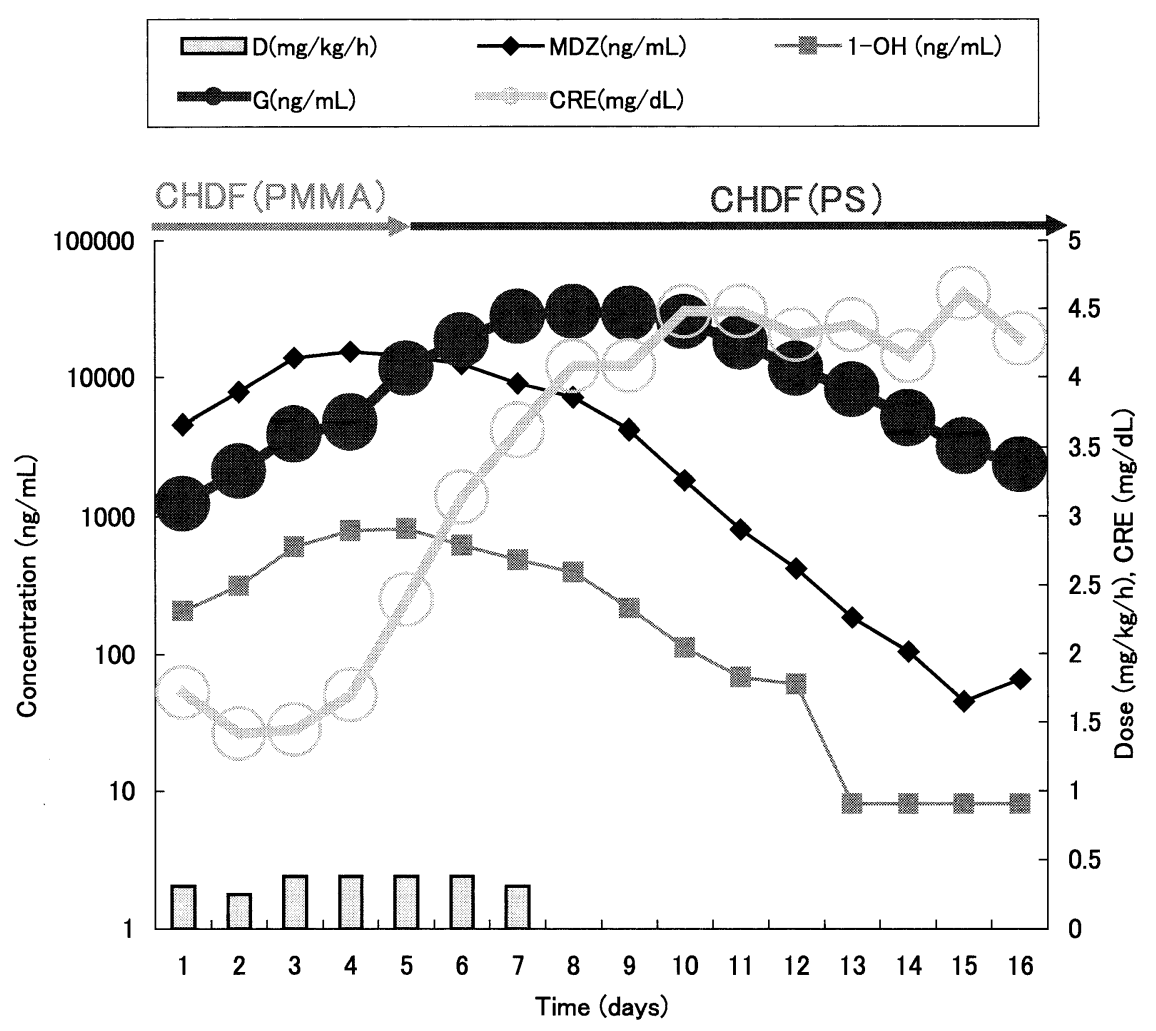

Fig. 6. Serum Midazolam and Active Metabolites Concentration-time Profiles and Clinical Data of Patient 7 Receiving CHDF (D=infusion rate, $\mathrm{MDZ}=$ midazolam, 1-OH $\mathrm{MDZ}=1$-hydroxymidazolam, $\mathrm{G}=1$-hydroxymidazolam glucuronide, $\mathrm{CRE}=$ serum creatinine). 
of creatinine in patients are indicative of renal functions, a significant positive correlation between the serum concentrations of creatinine and 1-hydroxymidazolam glucuronide in the patients receiving CHDF using PMMA was observed. It can be considered that CHDF using PMMA did not affect the elimination of 1-hydroxymidazolam glucuronide. In contrast, as shown in Fig. 4, we consider that CHDF using the PS membrane affects the elimination of 1-hydroxymidazolam glucuronide.

Extraction rate describes the capacity of a filter to remove a drug. The extraction rates obtained for midazolam and 1hydroxymidazolam suggest that CHDF is not effective in removing midazolam and 1-hydroxymidazolam from blood. This may be expected considering the high degree of protein binding of these compounds. On the other hand, 1-hydroxymidazolam glucuronide is effectively removed from blood by CHDF. The degree of binding of oxazepam glucuronides to serum albumin was found to be lower than that of the parent drug ${ }^{11}$. These results suggest that the glucuronidated form of 1-hydroxymidazolam exhibits a degree of protein binding lower than that of midazolam. However, despite CHDF using the PS membrane, we observed the accumulation of 1-hydroxymidazolam glucuronide in renal failure patients like patient 7. Such patients are sedated for several days due to increased 1-hydroxymidazolam glucuronide concentrations as a result of a reduced elimination of 1-hydroxymidazolam glucuronide. Bauer et al. ${ }^{12)}$ observed that the accumulation of 1-hydroxymidazolam glucuronide, which is a conjugated metabolite of midazolam, causes prolonged sedation in renal failure patients. Increased 1-hydroxymidazolam glucuronide concentrations can lead to unnecessarily long-term sedation. Accidentally prolonged sedation may also prolong the ICU stay and increase the risk of complications ${ }^{13)}$. In this study, the albumin concentrations of all patients were lower than the normal range. Decreased albumin concentration should be taken into consideration with an increased distribution volume and a decreased elimination of midazolam ${ }^{14)}$. Clinicians should take this into account to optimize the drug dose in patients receiving CHDF to avoid the accumulation of 1-hydroxymidazolam glucuronide and prolonged sedation. This seems to be especially important in patients with impaired renal function.

\section{References}

1) R. Bellomo, M. Farmer, G. Parkin, C. Wright, N. Boyce, Severe acute renal failure: A comparison of acute continuous hemodiafiltration and conventional dialytic therapy, Nephron, 71, 59-64 (1995).

2) E. van Bommel, N.D. Bouvy, K.L. So, R. Zietse, H.H. Vincent, H.A. Bruining, W. Weimar, Acute dialytic support for the critically ill : Intermittent hemodialysis versus continuous arteriovenous hemodiafiltration, Am. J. Nephrol, 15, 192-200 (1995).
3) P. Heizmann, M. Eckert, W.H. Ziegler, Pharmacokinetics and bioavailability of midazolam in man, Br. J. Clin. Pharmacol., 16, 43S-49S (1983).

4) J.W. Dundee, N.J. Halliday, K.W. Harper, R.N. Brogden, Midazolam. A review of its pharmacological properties and therapeutic use, Drugs, 28, 519-543 (1984).

5) A. Boukhabza, A.A. Lugnier, P. Kintz, P. Mangin, Simultaneous HPLC analysis of the hypnotic benzodiazepines nitrazepam, estazolam, flunitrazepam, and triazolam in plasma, J. Anal. Toxicol., 15, 319-322 (1991).

6) H. Ueno, S. Oda, N. Kitamura, T. Sadahiro, H. Shiga, T. Sugai, K. Naiki, S. Hikita, T. Hirano, N. Hirayama, Y. Hirayama, T. Hoshino, K. Matsuda, T. Moriguchi, K. Yokohari, E. Watanabe, "Theoretical consideration and practice of CHDF", ed by H. Hirasawa, Sougouigaku, Inc., Tokyo, 2000, pp.41-44.

7) U. Ebert, R. Oertel, W. Kirch, Physostigmine reversal of midazolam-induced electroencephalographic changes in healthy subjects, Clin. Pharmacol. Ther., 67, 538548 (2000).

8) B.K. Wagner, D.A. O'Hara, Pharmacokinetics and pharmacodynamics of sedatives and analgesics in the treatment of agitated critically ill patients, Clin. Pharmacokinet., 33, 426-453 (1997).

9) H. Oldenhof, M. de Jong, A. Steenhoek, R. Janknegt, Clinical pharmacokinetics of midazolam in intensive care patients, a wide interpatient variability?, Clin. Pharmacol. Ther., 43, 263-269 (1988).

10) M.T. Smith, M.J. Eadie, T.O. Brophy, The pharmacokinetics of midazolam in man, Eur. J. Clin. Pharmacol., 19, 271-278 (1981).

11) F.D. Boudinot, C.A. Homon, W.J. Jusko, H.W. Ruelius, Protein binding of oxazepam and its glucuronide conjugates to human albumin, Biochem Pharmacol., 34, 2115-2121 (1985).

12) T.M. Bauer, R. Ritz, C. Haberthur, H.R. Ha, W. Hunkeler, A.J. Sleight, G. Scollo-Lavizzari, W.E. Haefeli, Prolonged sedation due to accumulation of conjugated metabolites of midazolam, Lancet, 346, 145-147 (1995).

13) K. Hirata, Y. Matsumoto, A. Kurokawa, M. Onda, M. Shimizu, M. Fukuoka, M. Hirano and Y. Yamamoto, Possibility of influence of midazolam sedation on the diagnosis of brain death : concentrations of active metabolites after cessation of midazolam, YAKUGAKUZASSI, 123, 811-815 (2003).

14) T.B. Vree, M. Shimoda, J.J. Driessen, P.J. Guelen, T.J. Janssen, E.F. Termond, R. van Dalen, J.C. Hafkenscheid, M.S. Dirksen, Decreased plasma albumin concentration results in increased volume of distribution and decreased elimination of midazolam in intensive care patients, Clin. Pharmacol. Ther., 46, 537-544 (1989). 